\title{
LOCAL COMPLEMENTATION AND THE EXTENSION OF BILINEAR MAPPINGS
}

\author{
J.M.F. CASTILLO, A. DEFANT, R. GARCÍA, D. PÉREZ-GARCÍA, J. SUÁREZ
}

\begin{abstract}
We study different aspects of the connections between local theory of Banach spaces and the problem of the extension of bilinear forms from subspaces of Banach spaces. Among other results, we prove that if $X$ is not a Hilbert space then one may find a subspace of $X$ for which there is no AronBerner extension. We also obtain that the extension of bilinear forms from all the subspaces of a given $X$ forces such $X$ to contain no uniform copies of $\ell_{p}^{n}$ for $p \in[1,2)$. In particular, $X$ must have type $2-\varepsilon$ for every $\varepsilon>0$. Also, we show that the bilinear version of the Lindenstrauss-Pełczyński and Johnson-Zippin theorems fail. We will then consider the notion of locally $\alpha$-complemented subspace for a reasonable tensor norm $\alpha$, and study the connections between $\alpha$-local complementation and the extendability of $\alpha^{*}$-integral operators.
\end{abstract}

\section{INTRODUCTION}

The importance of the Hahn-Banach theorem in the linear theory of Banach spaces has encouraged the study of possible non-linear versions. Nevertheless, it is not difficult to see that there is no hope for a general Hahn-Banach result in this non-linear situation and the case of the scalar product in a Hilbert space is clear: this bilinear form can be extended from $\ell_{2}$ to a bigger superspace $X$ if and only if $\ell_{2}$ is complemented in $X$. The same happens to every Banach space isomorphic to its dual [13. There are however several interesting partial results. Curiously enough, there seem to be only two types of positive results: crude extension results and results involving linearity of the extension process. The two main sections of this paper, Sections 3 and 4, will study these two aspects of the extension problem for bilinear forms separately, as we describe now.

The general context for the problem is to take $Y$ a subspace of $X$ and consider the restriction operator $R: \mathcal{B}(X) \rightarrow \mathcal{B}(Y)$ between the spaces of bilinear (or, more generally, multilinear) continous forms.

(1) When is $R$ surjective? (i.e., when does there exist an extension, or else, a Hahn-Banach type theorem for bilinear forms?). The problem is considered in Section 3. Previous work can be found in $[12,13,8,17,26,30,35$. Very

2000 Mathematics Subject Classification. 46B20, 46B28, 46G20.

Key words and phrases. Hahn-Banach Extension theorems, bilinear forms, polynomials, Banach spaces.

The research of authors 1, 3 and 5 has been supported in part by MTM2010-20190-C02-01 and Junta de Extremadura CR10113 "IV Plan Regional I+D+i, Ayudas a Grupos de Investigación". The research of author 5 has been supported in part by a grant inside the network "Phenomena in high dimensions" at the University of Oldenburg under the supervision of professor A. Defant. 
recently, this question has been related to Bell inequalities in Quantum Information Theory [36]. Our results in this line nicely complement those in 22 .

(2) When does, moreover, $R$ admit a linear and continuous section? (i.,e when does there exist an Aron-Berner type extension theorem for bilinear forms?). This aspect of the problem is considered in Section 4 and connected with the complementation of the base spaces. Previous work in this direction can be found in $3,7,12,8,20,24,41$.

The paper is thus organized as follows: after this introduction and a "preliminaries" section, we treat in section 3 the possibility of extension theorems for bilinear forms. A combination of homological techniques and local theory of Banach spaces shows that most classical extension theorems for linear operators fail for bilinear forms: indeed, no Lindenstrauss-Pełczyński 31] or Johnson-Zippin 27] theorems remain valid in the bilinear setting. Contrarily to what occurs with Maurey's extension theorem [19: we obtain an analogue for bilinear forms and show that if all bilinear forms on subspaces of $X$ can be extended to $X$ then $X$ must have type $2-\varepsilon$ for every $\varepsilon>0$ and weak type 2 (see Theorem 11).

In section 4 we study the possibility of having linear continuous extension for bilinear forms, also called Aron-Berner extension. It is well known [12, 20, 41] that the existence of Aron-Berner extension for bilinear forms implies local complementation, which immediately implies, for instance, that the only subspaces of an $\mathcal{L}_{\infty}$ (resp. $\mathcal{L}_{1}$ ) space admitting Aron-Berner extension are those of the same type. Since the only space all whose subspaces are locally complemented is the Hilbert space, it follows that the only space for which there exists Aron-Berner extension for all its subspaces is the Hilbert space (see Theorem 3). We will extend the result for a finitely generated tensor norm (see Theorem 2).

In section 5, we study the local character of multi-linear extension with respect to a finitely generated tensor norm $\alpha$. We introduce the notion of local $\alpha$-extension (see definition 2) and characterize when an $\alpha$-polynomial (resp. multilinear) may be extended to an $\alpha$-polynomial (resp. multilinear) (see Theorems 4 and 5 ). Finally, we extend the notion of locally complemented subspace to a locally $\alpha$-complemented subspace, and show that it leads to the extendability of $\alpha^{*}$-integral operators (see Theorem 77).

\section{Notation and Preliminares}

In [16 the interested reader can find the most important tensor norms on tensor products of Banach spaces $\left(\varepsilon, \pi, \omega_{2}, \ldots\right)$. Given a Banach space $X$, we denote by $\operatorname{FIN}(X)$ or $\operatorname{COFIN}(X)$, respectively, the set of all isometric finite dimensional or finite codimensional closed subspaces of $X$.

Given a tensor norm $\alpha$ on $E_{1} \otimes \ldots \otimes E_{m}$, we say that $\alpha$ is finitely generated if for $z \in \otimes_{\alpha}^{m} E_{i}$ the norm can be computed as $\|z\|_{\otimes_{\alpha}^{m} E_{i}}=\inf \left\{\|z\|_{\otimes_{\alpha}^{m} M_{i}}: M_{i} \in\right.$ $\left.\operatorname{FIN}\left(E_{i}\right)\right\}$. 
Let $\alpha$ be a finitely generated tensor norm. We denote by $\mathcal{A}\left(E_{1}, \ldots, E_{m}\right):=$ $\left(\widetilde{\otimes}_{\alpha}^{m} E_{i}\right)^{*}$ the ideal of multi-linear maps associated to $\alpha$, see [23]. We denote the norm in this space by $\|\cdot\|_{\mathcal{A}}$. Under the natural identification, we will also refer to $\mathcal{A}\left(E_{1}, \ldots, E_{m}\right)$ as the space of $\alpha^{*}$-integral $m$-forms on $E_{1} \times \ldots \times E_{m}$, this is, the space of all $m$-linear maps $\varphi$ on $E_{1} \times \ldots \times E_{m}$ such that $L_{\varphi} \in \mathcal{A}\left(E_{1}, \ldots, E_{m}\right)$ endowed with the norm $\|\varphi\|_{\alpha^{*}}:=\left\|L_{\varphi}\right\|_{\mathcal{A}}$. We denote $\mathcal{A}_{m}(E)$ if $E=E_{i}$ for all $i$.

A Banach space $X$ is said to be an $\mathcal{L}_{p}$ for $1 \leq p \leq \infty$ if there exist a constant $\lambda>0$ such that for every finite dimensional subspace $E$ of $X$ there exists another finite dimensional subspace $F$ of $X$ with $F \supseteq E$ and $d\left(F, \ell_{p}^{\operatorname{dim} F}\right) \leq \lambda$.

A Banach space $X$ is said to have type $p$ (resp. cotype $q$ ) for $1 \leq p \leq 2$ (resp. $2 \leq q \leq \infty)$ if there is a constant $C$ such that for all finite subsets $x_{1}, \ldots, x_{n}$ of $X$

$$
\left\|\sum r_{i} x_{i}\right\|_{L_{2}[0,1]} \leq C\left(\sum\left\|x_{i}\right\|^{p}\right)^{1 / p}\left(\operatorname{resp} .\left(\sum\left\|x_{i}\right\|^{p}\right)^{1 / p} \leq C\left\|\sum r_{i} x_{i}\right\|_{L_{2}[0,1]}\right)
$$

where $r_{i}$ denotes the $i$-th Rademacher function. We recall that $\mathcal{L}_{p^{-}}$-spaces have type $\min \{p, 2\}$ and cotype $\max \{2, p\}$ and that a Banach space has both type 2 and cotype 2 if and only if it is isomorphic to a Hilbert space, see e.g. 38 .

A Banach space $X$ is said to have weak type 2 [39, p. 172] if there is a constant $C$ and a $\delta \in(0,1)$, so that whenever $E$ is a subspace of $X$ and an operator $T: E \rightarrow \ell_{2}^{n}$, there is an orthogonal projection $P$ on $\ell_{2}^{n}$ of rank $>\delta n$ and an operator $S: X \rightarrow \ell_{2}^{n}$ with

$$
S x=P T x \text { for all } x \in E \text {, and }\|S\| \leq C\|T\| .
$$

It is clear that type 2 implies weak type 2 , although the converse fails.

2.1. Locally complemented subspaces and short exact sequence. A short exact sequence of Banach spaces and linear continuous operators is a diagram

$$
0 \longrightarrow Y \stackrel{i}{\longrightarrow} X \stackrel{q}{\longrightarrow} Z \longrightarrow 0
$$

in which the image of each arrow coincides with the kernel of the following one. The open mapping theorem ensures that, given a sort exact sequence as before, $Y$ is a subspace of $X$ ( $i$ is an injection map) and $Z$ is the corresponding quotient $X / Y$ ( $q$ is a quotient map). The dual sequence

$$
0 \longrightarrow Z^{*} \stackrel{q^{*}}{\longrightarrow} X^{*} \stackrel{i^{*}}{\longrightarrow} Y^{*} \longrightarrow 0
$$

is also exact also. An exact sequence is said to split if $Y$ is complemented in $X$; which means that there is a linear continuous projection $p: X \rightarrow Y$. The sequence is said to locally split if the dual sequence splits.

Definition 1 (29]). Let $Y$ be a subspace of $X$ through a map $i$. We say that $Y$ is locally complemented in $X$ (through $i$ ) if there exists a constant $\lambda>0$ such that for every $F \in \mathrm{FIN}(X)$ there exist a retract $r_{F}: F \rightarrow Y$, this is $r_{F} i=i d$, with $\left\|r_{F}\right\| \leq \lambda$. For the quantitative version we will say locally $\lambda$-complemented. i.e.; the exact sequence $0 \rightarrow Y \rightarrow X \rightarrow Z \rightarrow 0 \lambda$-locally splits.

Kalton proved in 29] that the following conditions are equivalent:

(1) $Y$ is locally $\lambda$-complemented in $X$ through $i$

(2) $Y^{*}$ is $\lambda$-complemented in $X^{*}$ through $i^{*}$ 
(3) Every finite rank operator $T: Y \rightarrow F$ can be extended to $X$, say by $\widetilde{T}$ with $\|\widetilde{T}\| \leq \lambda\|T\|$.

It is therefore clear that $Y$ is locally $(\lambda)$ complemented in $X$ if and only if the exact sequence $0 \rightarrow Y \rightarrow X \rightarrow Z \rightarrow 0(\lambda)$ locally splits; and this happens if and only if the dual sequence $0 \rightarrow Z^{*} \rightarrow X^{*} \rightarrow Y^{*} \rightarrow 0(\lambda)$ splits.

We borrow from homological algebra the notation $\operatorname{Ext}(Z, Y)=0$ to mean that all exact sequences $0 \rightarrow Y \rightarrow X \rightarrow Z \rightarrow 0$ split. In [12] a homological approach was applied to the problem of extendibility of bilinear forms from $Y$ to $X$ to show:

- If $\operatorname{Ext}\left(Y,(X / Y)^{*}\right)=0$ and $\operatorname{Ext}\left(X / Y, X^{*}\right)=0$ then all bilinear forms on $Y$ extend to $X$.

- If $\operatorname{Ext}\left(X / Y, Y^{*}\right)=0$ and $\operatorname{Ext}\left(X,(X / Y)^{*}\right)=0$ then all bilinear forms on $Y$ extend to $X$.

This immediately implies that bilinear forms on $\mathcal{L}_{1}$ subspaces of $\mathcal{L}_{1}$ spaces extend to the bigger space (as well as bilinear form on subspaces of $\mathcal{L}_{\infty}$ spaces inducing $\mathcal{L}_{\infty}$-quotients) ([14, 29]). And all bilinear forms on $Y$ extend to $\mathcal{L}_{1}$ if only if $\operatorname{Ext}\left(Y,\left(\mathcal{L}_{1} / Y\right)^{*}\right)=0$, equivalently $\operatorname{Ext}\left(\mathcal{L}_{1} / Y, Y^{*}\right)=0($ see [12, Thm. 2.3 ] ).

\section{HAHN-BANACH TYPE EXTENSION THEOREMS FOR BILINEAR FORMS}

The problem of the extension of -even scalar!- bilinear forms is still mostly open. There is only a small number of papers $7,12,13,8,9,17,30$ in which a few techniques are developed.

Let us briefly recall the existing basic extension theorems for linear continuous operators: the Hahn-Banach theorem, the Lindenstrauss-Pełczyński theorem [31, the Johnson-Zippin theorem [27] and Maurey's theorem [19]. Are there analogues for bilinear forms?

3.1. Bilinear Lindenstrauss-Pełczyński theorem. The Lindenstrauss - Pełczyń ski theorem [31] establishes that every $C(K)$-valued operator defined on a subspace of $c_{0}$ can be extended to the whole $c_{0}$. Thus, can bilinear forms defined on subspaces of $c_{0}$ be extended to $c_{0}$ ? The answer is a sounding no. Let $\left(F_{n}\right)_{n}$ denote a sequence of finite dimensional Banach spaces uniformly isomorphic to their duals.

Proposition 1. Let $X$ be a Banach space containing a non-locally complemented subspace isomorphic to $c_{0}\left(F_{n}\right)$. There is a bilinear form on $c_{0}\left(F_{n}\right)$ that cannot be extended to $X$.

Proof. Observe that the hipothesis already implies $\lim \operatorname{dim} F_{n}=+\infty$ since $c_{0}$ is locally complemented in any superspace. Let, for each $n \in \mathbb{N}, \tau_{n}: F_{n} \rightarrow F_{n}^{*}$ be an isomorphism so that $\sup _{n}\left\|\tau_{n}\right\|\left\|\tau_{n}^{-1}\right\|=M<+\infty$. Since $c_{0}(\mathcal{F})$ is not locally complemented in $X$, the dual sequence

$$
0 \longrightarrow c_{0}\left(F_{n}\right)^{\perp} \longrightarrow X^{*} \longrightarrow \ell_{1}\left(F_{n}^{*}\right) \longrightarrow 0
$$

does not split, where $c_{0}\left(F_{n}\right)^{\perp}=\left(X / c_{0}\left(F_{n}\right)\right)^{*}$. This exactly means that any lifting of the canonical inclusion $j_{n}: F_{n}^{*} \rightarrow \ell_{1}\left(F_{n}^{*}\right)$ to $X^{*}$ must have norm greater than or 
equal to $g(n)$ with $\lim g(n)=+\infty$. Let $\sigma \in \ell_{1}$ be such that $\lim \sigma_{n} g(n)=+\infty$. We define the "diagonal" operator

$$
D_{\sigma}: c_{0}\left(F_{n}\right) \longrightarrow \ell_{1}\left(F_{n}^{*}\right)
$$

given by $D\left[\left(f_{n}\right)_{n}\right]=\left(\sigma_{n} \tau_{n} f_{n}\right)_{n}$. Let $J: c_{0}\left(F_{n}^{*}\right) \rightarrow c_{0}\left(F_{n}\right)$ be the isomorphism $J\left(g_{n}\right)=\left(\tau_{n}^{-1} g_{n}\right)$. The existence of a lifting $D^{\prime}: c_{0}\left(F_{n}\right) \rightarrow X^{*}$ for $D$ would imply the existence of a lifting $D^{\prime} J$ for $D J$ with norm at most $\left\|D^{\prime}\right\| M$. Thus, there are uniformly bounded liftings for the restrictions $D J_{\mid F_{n}^{*}}=\sigma_{n} j_{n}$, in contradiction with the assumption $\lim \sigma_{n} g(n)=+\infty$.

Corollary 1. Let $X$ be a Banach space containing uniform copies of $\ell_{2}^{n}$ not uniformly complemented. There are bilinear forms on some subspace of $c_{0}(X)$ that cannot be extended to the whole $c_{0}(X)$.

Proof. BY the hypothesis, $X$ contains copies $j_{n}: \ell_{2}^{n} \rightarrow X$, hence $c_{0}(X)$ contains $c_{0}\left(\ell_{2}^{n}\right)$ via the natural isomorphism $\left(j_{n}\right): c_{0}\left(\ell_{2}^{n}\right) \rightarrow c_{0}(X)$. If this $c_{0}\left(\ell_{2}^{n}\right)$ is locally complemented, the canonical projections $p_{n}: c_{0}\left(\ell_{2}^{n}\right) \rightarrow \ell_{2}^{n}$ could be uniformly extended to $c_{0}(X)$, yielding uniformly complemented copies of $\ell_{2}^{n}$, against the hypothesis.

An obvious corollary is:

Corollary 2. There is a bilinear form on a subspace of $c_{0}$ that cannot be extended to $c_{0}$.

This shows that no bilinear Lindenstrauss-Pełczyński theorem about the extension of $C(K)$-valued operators on subspaces of $c_{0}$ is possible.

Remarks. There are aspects of this problem not well understood. Observe that a subspace $Y$ of $c_{0}$ is locally complemented if and only if it is an $\mathcal{L}_{\infty}$-space, in which case [2] it must be isomorphic to $c_{0}$. Thus, $c_{0}\left(F_{n}\right)$ is not locally complemented in $c_{0}$ unless it is isomorphic to $c_{0}$; which implies that the spaces $F_{n}$ must be uniformly complemented in $c_{0}$, hence in some $\ell_{\infty}^{m}$. It is however a hard open problem, see 34, to determine if a sequence $F_{n}$ of finite dimensional Banach spaces uniformly complemented in $\ell_{\infty}^{m}$ must be uniformly isomorphic to $\ell_{\infty}^{k}$. On the other hand, it follows from [12 that if a subspace $Y$ of $c_{0}$ admits the extension of bilinear forms to $c_{0}$ then it is a Hilbert-Schmidt space, namely $\mathfrak{L}\left(Y, \ell_{2}\right)=\Pi_{2}\left(Y, \ell_{2}\right)$. For a subspace of the form $c_{0}\left(F_{n}\right)$ this condition implies that there is a uniform constant $C$ so that for every $n$ the $\pi_{2}$-summing norm and the standard operator norm are $C$-equivalent on $\mathfrak{L}\left(F_{n}, \ell_{2}^{n}\right)$. We do not know if this condition implies that the $F_{n}$ are uniformly complemented in $\ell_{\infty}^{m}$. All this together suggests:

The $c_{0}$-conjecture. A subspace of $c_{0}$ on which every bilinear form can be extended to the whole $c_{0}$ is isomorphic to $c_{0}$.

3.2. Bilinear Johnson-Zippin theorem. The Johnson-Zippin theorem [27] establishes that $\mathcal{L}_{\infty}$-valued operators defined on weak*- subspaces of $\ell_{1}$ can be extended to the whole $\ell_{1}$. Again, there is no bilinear Johnson-Zippin theorem. Indeed, one has

Proposition 2. Let $\left(F_{n}\right)$ be a sequence of finite dimensional Banach spaces that is dense, in the Banach-Mazur distance, in the family of all finite dimensional Banach 
spaces. If $H$ is any subspace of $c_{0}$ such that $H^{*}=\ell_{1}\left(F_{n}\right)$ then there are bilinear forms on $H^{\perp}$ that cannot be extended to $\ell_{1}$.

Proof. Standard facts from homological algebra (see [13], or else [11]) yield that for a subspace $A$ of $\ell_{1}$ the assertion "all operators $\mathfrak{L}(A, B)$ can be extended to $\ell_{1}$ " can be reformulated as $\operatorname{Ext}\left(\ell_{1} / A, B\right)=0$. Hence, given $H \subset c_{0}$ that all bilinear forms on $H^{\perp}$-i.e., operators from $\mathfrak{L}\left(H^{\perp}, H^{* *}\right)$ - can be extended to $\ell_{1}$ is equivalent to $\operatorname{Ext}\left(H^{*},\left(c_{0} / H\right)^{* *}\right)=0$, see [13, Theorem 2.3]. In the situation we are considering this means $\operatorname{Ext}\left(\ell_{1}\left(F_{n}\right),\left(c_{0} / H\right)^{* *}\right)=0$. But this means that $\operatorname{Ext}\left(F_{n},\left(c_{0} / H\right)^{* *}\right)=0$ uniformly on $n$-see [10]- from where it follows that $\left(c_{0} / H\right)^{* *}$ is an $\mathcal{L}_{\infty}$-space. But then also $c_{0} / H$ must be an $\mathcal{L}_{\infty}$-space, which would make $\ell_{1}\left(F_{n}\right)$ an $\mathcal{L}_{1}$-space, which is impossible.

An immediate corollary is:

Corollary 3. Every $\mathcal{L}_{1}$-space admits a bilinear form on a subspace that cannot be extended to the whole space.

This is somewhat surprising since every bilinear form on $\mathcal{L}_{1}$-spaces can be extended to $\mathcal{L}_{1}$-superspaces (see [13]). The surprise passes when one is shown that the case of $\ell_{1}$ is essentially the unique possible: it has been shown in [17 that if a subspace $Y$, with unconditional basis, of an $\mathcal{L}_{1}$-space is such that every $m$-linear form $\varphi$ on $Y$ extends to one $\widetilde{\varphi}$ on the whose space with $\|\widetilde{\varphi}\| \leq C^{m}\|\varphi\|$, then $Y=\ell_{1}$.

After this shock of negative results let us present a positive one. It is well known that Maurey's extension theorem admits a bilinear version so we provide a sort of converse.

\section{Theorem 1.}

(1) (Maurey) If $X$ has type 2 , then every bilinear form on a subspace of $X$ can be extended to the whole $X$.

(2) If every bilinear form on every subspace of $X$ can be extended to $X$ then $X$ must have type $2-\varepsilon$ for every $\varepsilon>0$.

Proof. It is clear that the first claim goes back to Maurey's extension Theorem [19, Corollary 12.23]. For the second, let us assume that there exists $p<2$ such that $\ell_{p}$ is finitely representable in $X$. Then $\ell_{p}^{n}$ is uniformly embedded in $X$ via $j_{n}: \ell_{p}^{n} \hookrightarrow X$. By the construction given in [4, Theorem 3.1] to obtain an un-complemented copy of $\ell_{2}$ in $L_{p}$ we can consider $T_{n}: \ell_{2}^{n} \rightarrow E_{n} \subset L_{p}$ to be uniformly bounded embeddings with no uniformly bounded projections. Moreover, for every $n$, there exist $(1+\varepsilon)$-embeddings $\Phi_{n}: E_{n} \rightarrow \ell_{p}^{k(n)} \subset L_{p}$, for $k(n)$ sufficiently large. So we have embeddings $i_{n}=\Phi_{n} \circ T_{n}: \ell_{2}^{n} \rightarrow \ell_{p}^{k(n)}$, that are uniformly bounded and with no uniformly bounded projections:

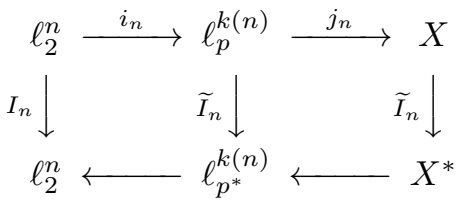


where $I_{n}: \ell_{2}^{n} \rightarrow \ell_{2}^{n}$ is the identity (Riesz-duality) associated to the bilinear scalar product. Since we can extend bilinear forms from subspaces of $X$, we can do it with a uniform constant (by the closed graph theorem). And this contradicts the fact that the $i_{n}$ 's do not have uniformly bounded projections. Since $\ell_{p}$ is not finitely representable for $p \in[1,2)$, by Maurey-Pisier Theorem [38, Theorem 3.11], $X$ has type $2-\varepsilon$ for every $\varepsilon>0$.

The result is somehow optimal since the converse to (2) fails: the Kalton-Peck space $Z_{2} 28$ has type $2-\varepsilon$ for every $\varepsilon>0$ and contains an uncomplemented copy of $\ell_{2}$. Consequently, the canonical bilinear form on $\ell_{2}$ cannot be extended to $Z_{2}$.

Nevertheless, we can still improve it a little turning in a different direction. Recall that a Banach space $X$ is said to en enjoy the Maurey extension property (MEP) if for every subspace $E \subset X$ all linear continuous operators $E \rightarrow \ell_{2}$ can be extended to operators $X \rightarrow \ell_{2}$. It is not to hard to show that MEP can be reformulated in finite dimensional terms: there is a constant $C>0$ such that for all finite dimensional subspaces $F \subset X$ all norm one operators $F \rightarrow \ell_{2}$ can be extended to operators $X \rightarrow \ell_{2}$ with norm at most $C$. Type 2 spaces have MEP, and a theorem of Milman and Pisier [33. Th. 10] establishes that $X$ must have weak type 2 . One has:

Proposition 3. If a Banach space $X$ is such that all bilinear forms on all subspaces can be extended to $X$ then $X$ has the Maurey extension property, hence it must have weak type 2.

Proof. The failure of MEP means the existence of a sequence $\left(F_{n}\right)$ of finite dimensional subspaces of $X$ and norm one operators $\phi_{n}: F_{n} \rightarrow \ell_{2}$ such that any extension of $\phi_{n}$ to $X$ has norm at least $g(n)$ with $\lim g(n)=+\infty$. Let $\sigma \in \ell_{1}$ such that $\lim \sigma_{n} g(n)=+\infty$. Standard techniques - see 34- allow us to assume that $\left(F_{n}\right)$ forms a finite-dimensional decomposition of its closed linear span $\Sigma F_{n}$. This subspace of $X$ admits the bilinear form $\Phi: \Sigma F_{n} \rightarrow\left(\Sigma F_{n}\right)^{*}$ defined as

$$
\Phi\left(\left(f_{n}\right)\right)\left(g_{n}\right)=\sum \sigma_{n}<\phi_{n} f_{n}, \phi_{n} g_{n}>
$$

which cannot be extended to $X$.

There are other situations in which it is possible to extend bilinear forms: such is the case of $\mathcal{L}_{\infty}$-spaces such as $A(\mathbb{D})$-the disk algebra-, $H^{\infty}$ or Pisier's spaces $P$ verifying $P \widehat{\otimes}_{\varepsilon} P=P \widehat{\otimes}_{\pi} P$. Actually, it was proved in [12, Theorem 2.2] that every bilinear form on $Y$ extends to any super-space iff $Y \widehat{\otimes}_{\omega_{2}} Y=Y \widehat{\otimes}_{\pi} Y$.

We conclude this section with a final remark. One additional obstruction to the possibility of obtaining extension theorems for bilinear forms is the following: bilinear forms are not, as a rule, weakly sequentially continuous (wsc, in short); and the scalar product in a Hilbert space is the perfect example. Nevertheless, all $C(K)$-spaces, as all spaces having the Dunford-Pettis property, have all bilinear forms wsc. Therefore the only chance to have an extension theorem for bilinear forms from a Banach space $X$ to some superspace $C(K)$ is that all bilinear forms on $X$ must be wsc. This conditions is however not enough, since all subspaces of $c_{0}$ have the Dunford-Pettis property and, as we have already shown, many of them 
admit bilinear forms that cannot be extended to $c_{0}$; the extension to bigger $C(K)$ spaces is impossible too by [12, Proposition 2.1]. On the positive side, bilinear forms on $A(\mathbb{D})$ or on $\mathcal{L}_{\infty}$-spaces can be extended to any superspace 12, Example $1]$.

\section{Linear CONTINUOUS EXTENSION OF BILINEAR FORMS}

The Aron-Berner 3 type extensions of bilinear forms is linear continuous extension and was shown to be equivalent to local complementation in [12, 20, 41.

Proposition 4. The following conditions are equivalent:

(1) $Y$ is locally complemented in $X$ through $j$.

(2) $Y \widehat{\otimes}_{\pi} Y$ is locally complemented in $X \widehat{\otimes}_{\pi} X$ (through $j \otimes j$ ).

(3) There exists an extension operator $\Phi_{2}: \mathcal{B}(Y) \rightarrow \mathcal{B}(X)$. i.e.; $\mathcal{B}(Y)$ is complemented in $\mathcal{B}(X)$.

(3) There exists an extension operator $\Phi_{n}: \mathcal{L}\left({ }^{n} Y\right) \rightarrow \mathcal{L}\left({ }^{n} X\right)$. i.e.; $\mathcal{L}\left({ }^{n} Y\right)$ is complemented in $\mathcal{L}\left({ }^{n} X\right)$.

In particular, since $\mathcal{L}_{\infty}$-spaces are locally complemented in every subspace, they always admit linear continuous extension of bilinear forms to any superspace. A partial positive answer to the $c_{0}$-conjecture can be presented.

Proposition 5. A subspace $Y$ of $c_{0}(\Gamma)$ admits the extension of bilinear forms to $c_{0}(\Gamma)$ in a linear and continuous form if and only if it is isomorphic to some $c_{0}(I)$. The same is true for n-linear maps.

Proof. By the proposition above, $Y$ must be locally complemented in $c_{0}(\Gamma)$, hence it must be an $\mathcal{L}_{\infty}$ space, and therefore [25], it must be isomorphic to some $c_{0}(I)$.

Proposition 4 easily extends to the multilinear setting.

Theorem 2. Let be $j: Y \hookrightarrow X$ and $\alpha$ a finitely generated $m$-tensor norm. The following conditions are equivalent:

(1) $Y$ is locally complemented in $X$ (through $j$ ).

(2) $\widetilde{\otimes}_{\alpha}^{m} Y$ is locally complemented in $\widetilde{\otimes}_{\alpha}^{m} X\left(\right.$ through $\left.\otimes_{\alpha}^{m} j\right)$

(3) $\mathcal{A}_{m}(Y)$ is complemented in $\mathcal{A}_{m}(X)$

Proof. (1) $\Rightarrow(2)$ Take $F \in \operatorname{FIN}\left(\otimes_{\alpha}^{m} X\right)$ and notice that $F \subseteq \otimes^{m} F_{i}$ with $F_{i} \in$ $\operatorname{FIN}(X)$. Consider for each $i$ a retraction $r_{F_{i}}: F_{i} \rightarrow Y$ and thus over $F$ we have the retraction $\otimes^{m} r_{F_{i}}$ (restricted to $\mathrm{F}$ ).

$(2) \Rightarrow(1)$ follows from the general fact: "if $Y$ is locally complemented in $X$ and $Y_{0} \subseteq Y, X_{0} \subseteq X$ are complemented subspaces such that $Y_{0} \subseteq X_{0}$ then $Y_{0}$ is locally complemented in $X_{0}$."

$(2) \Leftrightarrow(3)$ is exactly the equivalence $(1) \Leftrightarrow(2)$ in [29, Theorem. 3.5].

The proof of $(1) \Rightarrow(2)$ yields that when $Y$ is $\lambda$-locally complemented in $X$, then every $\alpha$-integral $m$-form $T$ on $Y$ can be extended to an $\alpha$-integral $m$-form $\widetilde{T}$ on $X$ with $\|\widetilde{T}\| \leq \lambda^{m+1}\|T\|$. This condition of extension with control of the norms was recently used in [17] and is the right condition to deal with extensions of holomorphic functions, see [17]. On the other hand taking an uncomplemented copy of $\ell_{1}$ inside $\ell_{1}$, see [5], one can see that this extension with control of the norms is not equivalent to local complementation. 
There is a partial converse to Proposition 4 which can be understood as the local version of the classical Lindentrauss-Tzafriri theorem [32. It essentially appears in Fakhoury [21, Thm. 3.7], implicitly in [1, Lemma 12.4.3]) and has been recently and independently obtained by Fernández Unzueta and Prieto [22, Thm. 2.1]. For the sake of completeness we include a clean draft for the proof.

Theorem 3. A Banach space $X$ is a Hilbert space if and only if every subspace is locally complemented .

Proof. For the non-trivial implication let us take an infinite dimensional space $X$ such that all of its subspaces are locally complemented. Let us define $\lambda_{X}(Y)$ as the infimum of the local complementation constants of $Y$ in $X$ and

$$
\lambda_{s}(X)=\sup \left\{\lambda_{X}(Y): Y \subset X\right\} .
$$

Assume that $\lambda_{s}(X)=\infty$. First, is clear that for every subspace $Y$ of finite codimension $\lambda_{s}(Y)=\infty$.

Let $E_{1}$ be a finite dimensional subspace of $X$ with $\lambda_{X}\left(E_{1}\right) \geq 1$. Using a standard argument a local theory (see, [15, Theorem 1] or [1, Lemma 12.4.3]) there is a finite codimensional subspace $Y_{1}$ such that the natural projection of $E_{1} \oplus Y_{1}$ to $E_{1}$ has norm $\leq 2$.

We now inductively construct a sequence of disjoint finite dimensional subspaces $\left(E_{n}\right)$ and finite codimensional subspaces $\left(Y_{n}\right)$ such that

$$
E_{n+1} \subset Y_{n} ; \quad Y_{n+1} \subset Y_{n}, \quad \lambda_{X}\left(E_{n}\right) \geq n
$$

and such that the projection constant from $E_{1} \oplus \cdots \oplus E_{n} \oplus Y_{n}$ to $E_{1} \oplus \cdots \oplus E_{n}$ has norm $\leq 2$. Let $Y$ be the following subspace of $X$ :

$$
Y=\left\{\sum e_{n}: e_{n} \in E_{n}\right.
$$

and $\sum e_{n}$ converges in $\left.X\right\} . Y$ is a subspace of $X$ and has the Schauder decomposition $\left(E_{n}\right)$. It is clear that the natural projection of $Y$ to $E_{n}$ has norm at most 4, which implies that $Y$ cannot be locally complemented in $X$.

All this together means that we have shown that $\lambda_{s}(X)<\infty$. In particular, this implies that there is a uniform constant $-\lambda_{s}(X)$ - co that all finite dimensional subspaces of $X$ are $\lambda_{s}(X)$-complemented in $X$. What remains is to follow the final step in the proof of the Lindenstrauss-Tzafriri classical theorem; see e.g. 1, Thm 12.4.2].

This characterization should be compared with the type of results in [21].

Corollary 4. There is no Banach space $X$ such that every infinite dimensional subspace is an $\mathcal{L}_{\infty}$-space.

Corollary 5. Given any Banach, non-Hilbert, space $X$ there always exist a weak*closed uncomplemented subspace of $X^{*}$.

\section{LOCALIZATION With RESPECT TO OTHER TENSOR NORMS}

It is part of the common believing that the extension of multi-linear forms is a local phenomenon. A different thing is to precise in which sense or senses this is true. Here we present a natural generalization of an idea presented in [12. We need to introduce some definitions.

By a normed ideal of $n$-linear continuous operators between Banach spaces (37]) we mean a pair $\left(\mathcal{A},\|\cdot\|_{\mathcal{A}}\right)$ such that 
(1) $\mathcal{A}\left(E_{1}, \ldots, E_{n} ; F\right)=\mathcal{A} \cap \mathcal{L}\left(E_{1}, \ldots, E_{n} ; F\right)$ is linear and $\|\cdot\|_{\mid \mathcal{A}\left(E_{1}, \ldots, E_{n} ; F\right)}$ is a norm.

(2) If $T_{j} \in \mathcal{L}\left(G_{j} ; E_{j}\right), \varphi \in \mathcal{A}\left(E_{1}, \ldots, E_{n} ; F\right)$ and $S \in \mathcal{L}(F ; G)$ then the composition $S \circ \varphi \circ\left(T_{1}, \ldots, T_{n}\right)$ is in $\mathcal{A}$ and $\left\|S \circ \varphi \circ\left(T_{1}, \ldots, T_{n}\right)\right\|_{\mathcal{A}} \leq\|S\|\|\varphi\|_{\mathcal{A}}\left\|T_{1}\right\| \ldots\left\|T_{n}\right\|$.

(3) The map $\left\{\mathbb{K}^{n} \ni\left(x_{1}, \ldots, x_{n}\right) \rightarrow x_{1} \cdots x_{n} \in \mathbb{K}\right\}$ belongs to $\mathcal{A}$ with corresponding $\mathcal{A}$-norm equal 1.

An ideal $\left(\mathcal{A},\|\cdot\|_{\mathcal{A}}\right)$ is called maximal if the condition $\|\varphi\|_{\mathcal{A}^{\max }}:=\sup \left\{\left\|Q_{L}^{F} \circ \varphi_{\mid M_{1} \times \ldots \times M_{n}}\right\|_{\mathcal{A}}: M_{j} \in \operatorname{FIN}\left(E_{j}\right), L \in \operatorname{COFIN}(F)\right\}<\infty$ implies that $\varphi \in \mathcal{A}$ and $\|\varphi\|_{\mathcal{A}}=\|\varphi\|_{\mathcal{A}^{\max }}$ holds (where $Q_{L}^{F}: F \rightarrow L$ is the projection). Given an ideal $\left(\mathcal{A},\|\cdot\|_{\mathcal{A}}\right)$, its maximal envelope is defined as the linear set $\mathcal{A}^{\max }:=\left\{\varphi \in \mathcal{M}\left(E_{1}, \ldots E_{n}\right):\|\varphi\|_{\mathcal{A}^{\max }}<\infty\right\}$ endowed with the norm $\|\cdot\|_{\mathcal{A}^{\max }}$.

A subclass $\mathcal{Q} \subset \mathcal{P}^{n}$ of $n$-homogeneous continuous scalar-valued polynomials on Banach spaces is called an ideal if

(1) $\mathcal{Q}(E)=\mathcal{P}^{n} \cap \mathcal{Q}$ is a linear subspace of $\mathcal{P}^{n}(E)$ for all Banach spaces $E$.

(2) If $T \in \mathcal{L}(E ; F), q \in \mathcal{Q}(F)$ then $q \circ T \in \mathcal{Q}$.

(3) The map $\left\{\mathbb{K} \ni z \rightarrow z^{n} \in \mathbb{K}\right\}$ belongs to $\mathcal{Q}$. If $\|\cdot\|_{\mathcal{Q}}: \mathcal{Q} \rightarrow[0, \infty]$ satisfies

(4) $\left.\|\cdot\|_{\mathcal{Q}}\right|_{\mathcal{Q}(E)}$ is a norm for all Banach spaces $E$.

(5) $\|q \circ T\|_{\mathcal{Q}} \leq\|T\|^{n}\|q\|_{\mathcal{Q}}$ in the situation of (2).

(6) $\left\|\left\{\mathbb{K} \ni z \rightarrow z^{n} \in \mathbb{K}\right\}\right\|_{\mathcal{Q}}=1$,

then $\left(\mathcal{Q},\|\cdot\|_{\mathcal{Q}}\right)$ is called a normed ideal of $n$-homogeneous polynomials. For $\left(\mathcal{Q},\|\cdot\|_{\mathcal{Q}}\right)$ and $q \in \mathcal{P}^{n}(E)$ define $\|q\|_{Q_{\max }}:=\sup \left\{\left\|\left.q\right|_{M}\right\|_{\mathcal{Q}} \mid M \in \operatorname{FIN}(E)\right\} \in[0, \infty]$. $\left(\mathcal{Q},\|\cdot\|_{\mathcal{Q}}\right)$ is called maximal if every $q \in \mathcal{P}^{n}(E)$ with $\|q\|_{Q_{\max }}<\infty$ is in $\mathcal{Q}$ and $\|q\|_{Q}=\|q\|_{Q_{\max }}$. Given an ideal $\left(\mathcal{Q},\|\cdot\|_{\mathcal{Q}}\right)$, we define its maximal envelope, as before, like $\mathcal{Q}^{\max }:=\left\{q \in \mathcal{P}^{n}(E):\|q\|_{\mathcal{Q}_{\max }}<\infty\right\}$ endowed with the norm $\|\cdot\|_{\mathcal{Q}_{\max }} \cdot\left(\mathcal{Q},\|\cdot\|_{\mathcal{Q}}\right)$ is called ultrastable if for every ultrafilter $\mathfrak{U}$ the following holds: For $q_{\iota} \in \mathcal{Q}\left(E_{\iota}\right)$ with $\sup _{\iota \in I}\left\|q_{\iota}\right\|_{\mathcal{Q}}<\infty$ one has $\lim _{\mathfrak{U}} q_{\iota} \in \mathcal{Q}\left(\left(E_{\iota}\right)_{\mathfrak{U}}\right)$ and $\left\|\lim _{\mathfrak{U}} q_{\iota}\right\|_{\mathcal{Q}} \leq \sup \left\|q_{\iota}\right\|_{\mathcal{Q}}$ (and hence $\leq \lim _{\mathfrak{U}}\left\|q_{\iota}\right\|_{\mathcal{Q}}$ ).

Definition 2. Let $j_{i}: Y_{i} \rightarrow X_{i}$ be embeddings, $i=1, \ldots, n$, and $\alpha$ a finitely generated tensor norm. We say that the multi-linear mapping $\varphi: \prod Y_{i} \rightarrow \mathbb{K}$ admits a local $\alpha$-extension to $\prod X_{i}$ through $\prod j_{i}$ if there exists a constant $\lambda$ such that $\forall E_{i} \in \operatorname{FIN}\left(Y_{i}\right), F_{i} \in \operatorname{FIN}\left(X_{i}\right)$ we have that, for $i_{E}: \prod E_{i} \rightarrow \prod Y_{i}, \varphi i_{E}: \prod E_{i} \rightarrow \mathbb{K}$ admits an extension $\widetilde{\varphi}: \prod\left(E_{i}+F_{i}\right) \rightarrow \mathbb{K}$ through $\left(j_{i}\right)_{i=1}^{m}$ with $\|\widetilde{\varphi}\|_{\alpha^{*}} \leq \lambda\left\|\varphi i_{E}\right\|_{\alpha^{*}}$. Equivalently, $L_{\varphi i_{E}} \in\left(\widetilde{\otimes}_{\alpha}^{m} E_{i}\right)^{*}$ admits an extension $L_{\widetilde{\varphi}} \in\left(\widetilde{\otimes}_{\alpha}^{m}\left(E_{i}+F_{i}\right)\right)^{*}$ with $\left\|L_{\widetilde{\varphi}}\right\|_{\mathcal{A}} \leq \lambda\left\|L_{\varphi i_{E}}\right\|_{\mathcal{A}}$.

For the quantitative version we shall say $\varphi$ admits a local $(\alpha, \lambda)$-extension. As in 12 local extension gives full extension.

Theorem 4. Let $j_{i}: Y_{i} \hookrightarrow X_{i}$ be an isometric embedding for $i=1, \ldots, m$ and $\alpha$ a finitely generated tensor norm. For a given multilinear map $\varphi: \prod Y_{i} \rightarrow \mathbb{K}$ with $\|\varphi\|_{\alpha^{*}}<\infty$, the following conditions are equivalent:

(1) $\varphi$ admits a local $(\alpha, \lambda)$-extension to $\prod X_{i}$.

(2) $\varphi$ admits a full extension to $\prod X_{i}$, say $\widetilde{\varphi}$, with $\|\widetilde{\varphi}\|_{\alpha^{*}} \leq \lambda\|\varphi\|_{\alpha^{*}}$.

Proof. For each $i \in\{1, \ldots, m\}$ take an ultrafilter $\mathfrak{U}_{i}$ dominating the filter induced by the natural order on the couples $\left(E_{i}, F_{i}\right)$ with $E_{i} \in \operatorname{FIN}\left(Y_{i}\right), F_{i} \in \operatorname{FIN}\left(X_{i}\right)$ and 
consider the ultrafilter $\mathfrak{U}=\prod_{i=1}^{m} \mathfrak{U}_{i}$ on $I:=\prod_{i=1}^{m}\left(\operatorname{FIN}\left(Y_{i}\right), \operatorname{FIN}\left(X_{i}\right)\right)$. Thanks to Theorem 3.4 in 23] we know that the ideal $\mathcal{A}\left(Y_{1}, \ldots, Y_{m}\right):=\left(\widetilde{\otimes}_{\alpha}^{m} Y_{i}\right)^{*}$ is maximal and ultrastable. For our fixed $\varphi: \prod Y_{i} \rightarrow \mathbb{K}$ and for each $\iota \in I, \iota=$ $\prod_{i=1}^{m}\left(E_{i}, F_{i}\right)$, we have that $\varphi i_{E}$, where $i_{E}: \prod E_{i} \hookrightarrow \prod Y_{i}$ is the natural embedding, admits an extension $\varphi_{\iota}: \prod_{i=1}^{m}\left(E_{i}+F_{i}\right) \rightarrow \mathbb{K}$ such that $\varphi_{\iota} \in \mathcal{A}$ and $\left\|\varphi_{\iota}\right\|_{\alpha^{*}} \leq \lambda\left\|\varphi i_{E}\right\|_{\alpha^{*}} \leq \lambda\|\varphi\|_{\alpha^{*}}$. Obviously $\sup _{\iota \in I}\left\|\varphi_{\iota}\right\|_{\alpha^{*}} \leq \lambda\|\varphi\|_{\alpha^{*}}<\infty$ (and consequently $\sup _{\iota \in I}\left\|L_{\varphi_{\iota}}\right\|_{\mathcal{A}} \leq \lambda\left\|L_{\varphi}\right\|_{\mathcal{A}}<\infty$ ). By the ultrastability of $\mathcal{A}$, the map

$$
{\overline{\left(\varphi_{\iota}\right.}}_{\mathfrak{U}}\left(\prod_{i=1}^{m}\left(x_{\iota}^{i}\right)_{\mathfrak{U}_{i}}\right):=\left(\varphi_{\iota}\left(x_{\iota}^{1}, \ldots, x_{\iota}^{m}\right)\right)_{\mathfrak{U}}=\lim _{\mathfrak{U}} \varphi_{\iota}\left(x_{\iota}^{1}, \ldots, x_{\iota}^{m}\right)
$$

defines an $m$-linear map $\prod_{i=1}^{n}\left(E_{i, \iota}+F_{i, \iota}\right)_{\mathfrak{U}_{i}} \rightarrow \mathbb{K}$ such that

$$
\left\|L_{\overline{\left(\varphi_{\iota}\right)_{\mathfrak{U}}}}\right\|_{\mathcal{A}} \leq \lim _{\mathfrak{U}}\left\|L_{\varphi_{\iota}}\right\|_{\mathcal{A}} \leq \lambda\left\|L_{\varphi}\right\|_{\mathcal{A}}
$$

and hence

$$
L \overline{\left(\varphi_{\iota}\right)_{\mathfrak{U}}} \in \mathcal{A}\left(\left(E_{1, \iota}+F_{1, \iota}\right)_{\mathfrak{H}_{1}}, \ldots,\left(E_{n, \iota}+F_{n, \iota}\right)_{\mathfrak{U}_{n}}\right)=\left(\widetilde{\otimes}_{\alpha}^{m}\left(E_{i, \iota}+F_{i, \iota}\right)_{\mathfrak{H}_{i}}\right)^{*} .
$$

The rest is to convince us that we have the chain of embedding $\prod Y_{i} \hookrightarrow \prod X_{i} \hookrightarrow$ $\prod\left(E_{i, \iota}+F_{i, \iota}\right)_{\mathfrak{U}_{i}}$. To this end for a point $\left(y_{i}\right)_{i=1}^{m} \in \prod Y_{i}$ and $\iota=\prod\left(E_{i}, F_{i}\right)$ we define

$$
y_{i, \iota}:= \begin{cases}j_{i}\left(y_{i}\right), & \text { if } y_{i} \in E_{i} \\ 0, & \text { otherwise. }\end{cases}
$$

It follows that

$$
J: \prod_{\left(y_{i}\right) \rightarrow\left(y_{1, \iota}, \ldots, y_{m, \iota}\right)_{\iota}}\left(\prod_{i, \iota}+F_{i, \iota}\right)_{\mathfrak{U}_{i}}
$$

is an isometric embedding by the definition of the norm in the ultraproduct. The same arguments for $\prod X_{i}$, defining

$$
x_{i, \iota}:= \begin{cases}x_{i}, & \text { if } x_{i} \in F_{i} \\ 0, & \text { otherwise }\end{cases}
$$

allow us to get the corresponding isometric embedding $I: \prod X_{i} \hookrightarrow \prod\left(E_{i, \iota}+F_{i, \iota}\right)_{\mathfrak{U}_{i}}$. It is routine to check that $I \circ\left(j_{i}\right)_{i=1}^{m}=J$ and thus $\widetilde{\varphi}:={\overline{\left(\varphi_{\iota}\right)_{\mathfrak{U}}}} \circ I$ is the required extension of $\varphi$.

The notion of locally $\alpha$-extension admits an obviously symmetric form so it is also possible the "polynomial version" of Theorem 4. This "polynomial version" in terms of operator ideals works as:

Theorem 5. Let $\mathcal{Q}(\cdot)$ be an ideal of $m$-homogeneous polynomials and consider an embedding $j: Y \hookrightarrow X$. If a given $q \in \mathcal{Q}(Y)$ admits a symmetric local extension to $\mathcal{Q}(X)$ then there exists an extension $\widetilde{q} \in \mathcal{Q}^{\max }(X)$.

Proof. The main ingredient of the proof is the representation theorem of [23. Given the operator ideal $\mathcal{Q}$ we define a tensor norm on finite-dimensional spaces, as usual, by $\otimes_{\alpha_{s}}^{m} M:=\mathcal{Q}(M)^{*}$ and extend to all normed spaces $Z$ by

$$
\|z\|_{\otimes_{\alpha_{s}}^{m} Z}:=\inf \left\{\|z\|_{\otimes_{\alpha_{s}}^{m} M} \mid M \in \operatorname{FIN}(Z), z \in \otimes_{s}^{m} Z\right\} .
$$

Now, for a given $q \in \mathcal{Q}(Y)$, we restrict ourselves to the finite-dimensional subspaces of $Y$ and $X$. This is, $\forall E \in \operatorname{FIN}(Y), F \in \operatorname{FIN}(X)$ we know that $q \in\left(\otimes_{\alpha_{s}}^{m} E\right)^{*}=$ 
$\mathcal{Q}(E)^{* *}=\mathcal{Q}(E)$ admits an extension to $\widehat{q} \in\left(\otimes_{\alpha_{s}}^{m}(E+F)\right)^{*}=\mathcal{Q}(E+F)^{* *}=$ $\mathcal{Q}(E+F)$ with $\sup \|\widehat{q}\|_{\mathcal{Q}}<\infty$. Similar arguments as in the Theorem 4, see 23, provide us a full extension $\widetilde{q} \in\left(\otimes_{\alpha_{s}}^{m} X\right)^{*}$ and using the representation theorem of [23] we have that $\left(\otimes_{\alpha_{s}}^{m} X\right)^{*}=\mathcal{Q}^{\max }(X)$.

The preceding result admits also a formulation for multi-linear operator ideals:

Theorem 6. Let $\mathcal{A}$ be an ideal of m-linear forms and consider embedding $j_{i}$ : $Y_{i} \hookrightarrow X_{i}$ for $i=1, \ldots, m$. If a given $\varphi \in \mathcal{A}\left(Y_{1}, \ldots, Y_{m}\right)$ admits a local extension to $\mathcal{A}\left(X_{1}, \ldots, X_{m}\right)$ then there exists a full extension $\widetilde{\varphi} \in \mathcal{A}^{\max }\left(X_{1}, \ldots, X_{m}\right)$.

We define now local $\alpha$-complementation.

Definition 3 (Local $\alpha$-complementation). Let $j: Y \hookrightarrow X$ be an embedding and $\alpha$ a tensor norm. We say that $Y$ is locally $\alpha$-complemented in $X$ through $j$ if there exists a constant $\lambda$ such that for every $F \in \operatorname{FIN}(X)$, every operator $T: Y \rightarrow F^{*}$ can be extended to $X$ by an operator $\widetilde{T}: X \rightarrow F^{*}$ with estimate $\|\widetilde{T}\|_{\mathcal{A}} \leq \lambda\|T\|_{\mathcal{A}}$.

One has:

- Every space is locally $\varepsilon$-complemented in any superspace.

- Local $\pi$-complementation is a weaker notion than classical local complementation; i.e., local complementation implies local $\pi$-complementation. Thus, every Banach space is locally $\pi$-complemented in its bidual and every $\mathcal{L}_{\infty}$-space is locally $\pi$-complemented in any superspace. The converse does not hold: take an uncomplemented subspace $Y$ of $L_{p}$, for $2<p<\infty$. Clearly, any $M \in \operatorname{FIN}\left(L_{p}\right)$ verifies that $C_{2}\left(M^{*}\right) \leq T_{2}\left(L_{p}\right)$, see [19]. By Maurey's extension theorem we can extend any operator $Y \rightarrow M^{*}$ to $L_{p}$ with the norm of the extension controlled by a universal constant. Hence $Y$ is locally $\pi$-complemented in $L_{p}$ but it cannot be locally complemented because, being $L_{p}$ is reflexive, it would be complemented.

Moreover, if $X$ contains all finite dimensional Banach spaces, e.g. $X=$ $C[0,1]$, then $X$ is locally complemented in $E$ if and only if $X$ is locally $\pi$-complemented in $E$.

- Let $\omega_{2}$ be the tensor norm associated to the $\gamma_{2}$ norm for finite rank operators defined as

$$
\gamma_{2}(T)=\inf \left\|T_{1}\right\|\left\|T_{2}\right\|
$$

where the inf is taken over all possible factorizations $T=T_{1} T_{2}=T$ of $T$ through a Hilbert space. As a consequence of Maurey's extension theorem one gets that every subspace $Y$ of a type 2 Banach space $X$ is locally $\omega_{2}$-complemented in $X$ with constant at most $k_{G} T_{2}(X) C_{2}\left(X^{*}\right)$, see [19].

Definition 4. We say that every $\alpha^{*}$-integral bilinear form on $Y$, a subspace of $X$, separately extends to $X$ if the following two conditions hold:

(1) Every $\alpha^{*}$-integral bilinear form on $Y \times Y$ extends to an $\alpha^{*}$-integral bilinear form on $Y \times X$.

(2) Every $\alpha^{*}$-integral bilinear form on $Y \times X$ extends to an $\alpha^{*}$-integral bilinear form on $X \times X$.

We are ready to connect the extension of bilinear forms with the notion of local $\alpha$-complementation. 
Theorem 7. Let $\alpha$ be a finitely generated tensor norm. If $Y$ is locally $\alpha$-complemented in $X$ then every $\alpha^{*}$-integral bilinear form on $Y$ extends separately to $X$.

Proof. Given a subspace $M \in \mathrm{FIN}(Y) \subset \mathrm{FIN}(X)$, we can construct the following diagram

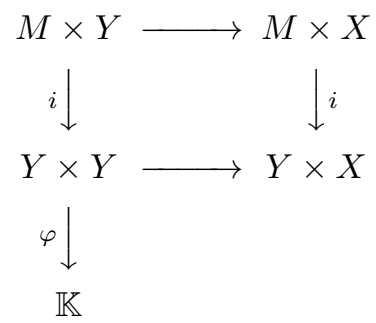

By hypothesis $\left.\varphi\right|_{M \times Y}$ extends to $M \times X$ for all $M \in \operatorname{FIN}(Y)$ and it is therefore enough to apply Theorem 4 . That every bilinear form on $Y \times X$ extends to $X \times X$ follows the same arguments. Notice that if $Y$ is locally $\alpha$-complemented in $X$ with constant $\lambda$, then we get $\lambda^{2}$ as extension constant for bilinear forms.

\section{REFERENCES}

[1] F. Albiac and N. J. Kalton, Topics in Banach Space Theory, vol. 233 of Graduate Texts in Mathematics, Springer, New York, NY, USA, 2006.

[2] D.E. Alspach, Quotients of $c_{0}$ are almost isometric to subspaces of $c_{0}$, Proc. Amer. Math. Soc. 76 (1979) 285-289.

[3] R. Aron and P. Berner. A Hahn-Banach extension theorem for analytic mappings, Bull. Soc. Math. France 106 (1978), 3-24.

[4] G. Bennett, L.E. Dor, V. Goodman and W.B. Johnson. On uncomplemented subspaces of $L_{p}, 1<p<2$, Israel J. Math. 26 no. 2 (1977), 178-187.

[5] J. Bourgain. A counterexample to a complementation problem, Compo. Math. 43 (1981), 133-144.

[6] F. Cabello Sánchez and J.M.F. Castillo, Uniform boundedness and twisted sums of Banach spaces, Houston J. Math. 30 (2004) 523-536 .

[7] F. Cabello Sánchez, R. García and I. Villanueva. Extensions of multilinear operator on Banach spaces, Extracta Math. 15 (2000), 291-334.

[8] D. Carando. Extendible polynomials on Banach spaces, J. Math. Anal. Appl. 233 (1999), 359-372.

[9] D. Carando and V. Dimant. Extension of polynomials and John's theorem for symmetric tensor products, Proc. Amer. Math. Soc. 135 (2007), 1769-1773.

[10] J.M.F. Castillo, Snarked sums of Banach spaces, Extracta Math. 12 (1997) 117-128.

[11] J.M.F. Castillo, Banach spaces, à la recherche du temps perdu, Extracta Math. 15 (2000) 373-390.

[12] J.M.F. Castillo, R. García and J.A. Jaramillo. Extension of bilinear forms on Banach spaces, Proc. Amer. Math. Soc. 129 (2001), 647-656.

[13] J.M.F. Castillo, R. García and J.A. Jaramillo. Extension of bilinear forms from subspaces of $\mathcal{L}_{1}$-spaces, Ann. Acad. Scientiarum Fennicae 27 (2002), 91-96.

[14] J.M.F. Castillo, R. García and J. Suárez Extension and lifting of operators and polynomials, Mediterranean J. Math. (to appear).

[15] W.J. Davis, D.W. Dean and I. Singer. Complemented subspaces and $\Lambda$ systems in Banach spaces, Israel J. Math. 6 (1968), 303-309.

[16] A. Defant and K. Floret. Tensor norms and Operator ideals, North-Holland Math. Stud. 176, 1993.

[17] A. Defant, D. García, M. Maestre and D. Pérez-García. Extension of multilinear forms and polynomials from subspaces of $\mathcal{L}_{1}$-spaces, Houston J. Math. 33 (2007), no. 3, 839-860.

[18] A. Defant, M. Maestre, and P. Sevilla-Peris. Cotype 2 estimates for spaces of polynomials on sequence spaces., Israel J. Math. 129 (2002), 291-315 .

[19] J. Diestel, H. Jarchow and A. Tonge. Absolutely Summing Operators, Cambridge Univ. Press, 1995.

[20] S. Dineen. Complex Analysis on Infinite Dimensional Spaces, Monographs in Math., Berlin 1999. 
[21] H. Fakhoury. Sélections linéaires associées au théorème de Hahn-Banach, J. Funct. Anal. 11 (1972), 436?-452.

[22] M. Fernández Unzueta and A. Prieto, Extension of polynomials defined on subspaces, Math. Proc. Cambridge Philos. Soc. 148 (2010) 505-518.

[23] K. Floret and S. Hunfeld. Ultrastability of ideals of homogeneous polynomials and multilinear mappings on Banach spaces, Proc. Amer. Math. Soc. 130, No.5, 1425-1435.

[24] P. Galindo, D. García, M. Maestre and J. Mujica. Extension of multilinear mappings on Banach spaces, Studia Math. 108 (1994), 55-76.

[25] G. Godefroy, N.J. Kalton and G. Lancien, Subspaces of $c_{0} \mathbb{N}$ ) and Lipschitz isomorphisms, Geometric and Functional Analysis 10 (2000) 798-820.

[26] H. Jarchow, C. Palazuelos, D. Pérez-García and I. Villanueva. Non-linear Hahn-Banach extensions and summability, J. Math. Anal. Appl. 336 (2007), 1161-1177.

[27] W.B. Johnson and M. Zippin. Extension of operator from weak-closed subspaces of $\ell_{1}$ an $C(K)$ spaces, Studia Math. 117 (1995), 43-55.

[28] N.J. Kalton and N.T. Peck. Twisted sums of sequence spaces and the three space problem, Trans. Amer. Math. Soc. 255 (1979), 1-30.

[29] N.J. Kalton. Locally complemented subspaces and $\mathcal{L}_{p}$-spaces for $0<p<1$, Math. Nachr. 115 (1984), 71-97.

[30] P. Kirwan and R. Ryan. Extendiblity of homogeneous polynomials on Banach spaces, Proc. Amer. Math. Soc. 126 (1998), 1023-1029.

[31] J. Lindenstrauss and A. Pełczyński. Contributions to the theory of classical Banach spaces, J. Funct. Anal. 8 (1971), 225-249.

[32] J. Lindenstrauss and L. Tzafriri. On the complemented subspaces problem, Israel J. Math. 9 (1971), 263-269.

[33] V.D. Milman and G. Pisier, Banach spaces with a weak cotype 2 property, Israel J. Math. 54 (1986) 139-158.

[34] Y. Moreno and A. Plichko. On automorphic Banach spaces, Israel J. of Math. 169 (2009) $29-47$.

[35] D. Pérez-García. A counterexample using 4-linear forms, Bull. Austral. Math. Soc. 70 (2004) 469-473.

[36] D. Pérez-García, M.M. Wolf, C. Palazuelos, I. Villanueva and M. Junge. Quantum Bell inequalities can have arbitrarily large violation, Commun. Math. Phys. 279 (2008), 455486 .

[37] A. Pietsch. Ideals of multilinear functionals, Proc. 2 Int. Conf. Operator Alg., Ideals and Their Applications in Theoretical Physics, 185199, Teubner-Texte, Leipzig, 1983.

[38] G. Pisier. Factorization of linear operators and geometry of Banch spaces, CBMS 60.

[39] G. Pisier, The volume of convex bodies and Banach space geometry, Cambridge Tracts in Math. 94, Cambridge Univ. Press, Cambridge, 1989.

[40] N. Tomczak-Jaegermann. Banach-Mazur Distances and Finite-Dimensional Operator Ideals, Longman Scientific Technical, 1989.

[41] I. Zalduendo. Extending polynomials - a survey, Publicaciones del Departamento de Análisis Matemático de la Univ. Complutense de Madrid, 41, 1998.

Departamento de Matemáticas, Facultad de Ciencias, Univ. de Extremadura, Avda. De Elvas S/N, 06071 Badajoz (Spain).

E-mail address: castillo@unex.es

E-mail address: rgarcia@unex.es

Institüt fur Mathematik, Universität Oldenburg, Postfach 2503, 26111 Oldenburg (GERMANY).

E-mail address: defant@mathematik.uni-oldenburg.de

Departamento de Análisis Matemático, Facultad de CC Matemáticas, Univ. Complutense de Madrid, Pza. de Ciencias 3, Ciudad Universitaria, 28040 Madrid (Spain).

E-mail address: dperez@mat.ucm.es

Escuela Politécnica, Universidad de Extremadura, Avenida de la Universidad s/n, 10071 CÁCERes, SPAin.

E-mail address: jesus@unex.es 
